\title{
Compressed sensing cardiac MRI exploiting spatio-temporal sparsity
}

\author{
Jafar Zamani ${ }^{1}$, Abbas N Moghaddam ${ }^{1,2^{*}}$, Hamidreza Saligheh Rad ${ }^{3,4}$ \\ From 16th Annual SCMR Scientific Sessions \\ San Francisco, CA, USA. 31 January - 3 February 2013
}

\section{Background}

Compressed Sensing (CS) is a theory with potential to reconstruct sparse images from a small number of random acquisitions. Particularly in MRI, CS aims to reconstruct the image from incomplete $\mathrm{K}$-space data with minimum penalty on the image quality. The image is recovered from the sub-sampled K-space data, using image sparsity in a known sparse transform domain. Cardiac MRI has a sparse structure in both temporal and spatial domains; making CS a promising method for such application.

\section{Methods}

Experiments were performed on a data set acquired by Cagdas Bilen et al.[1]. Fully sampled data were acquired using a $128 \times 128$ matrix $(\mathrm{FOV}=320 \times 320 \mathrm{~mm})$ and 23 temporal frames covering the cardiac cycle. In this study, we reconstructed eight (one in every three) frames through CS using Gradient Projection for Sparse Reconstruction (GPSR) algorithm. The remaining 15 frames were reconstructed through a combination of CS and temporal information (TI). Sampling rate for the CS and CS-TI frames was set to 0.5 and 0.3 , respectively. Block Discrete Cosine Transform (BDCT), Block WalshHadamard Transform (BWHT) and Gaussian Transform were used to create measurement matrix in CS. Discrete Wavelet Transform (DWT) was used as sparse basis. The fidelity term in cost function is modified as: $g=0.9 \|$
Fu $\mathrm{m}-\mathrm{y}\|2+0.1\| \mathrm{TE}-\mathrm{m} \| 2$, where $\mathrm{Fu}$ represents the under-sampled Fourier operator, y represents the Kspace under-sampled data, and TE (Temporal Estimation) represents the obtained frames from TI. In this study, we use interpolation (I), forward motion estimation (FME) and forward-backward motion estimation (F-B ME), respectively on previous and next CS frames to obtain TI.

\section{Results}

Figure 1 illustrates one frame from the original set along with the corresponding CS and CS-TI frames reconstructed with proposed methods for TI generation. Table 1 shows numerical results including SNR, PSNR, Structural SIMilarity (SSIM) and computational time for each proposed method.

\section{Conclusions}

The proposed method increased under-sampling rate and expedited reconstruction time in CS theory. The results were quantified using SNR, PSNR and SSIM for the quality of the reconstruction and the computational time, concluding that BWHT outperforms other methods in both quality measures and computational time with $15 \%$ and $10 \%$, respectively. In all aforementioned a derivative of the proposed method, the processing time was at least 4 times accelerated compared to the routine CS algorithm. 


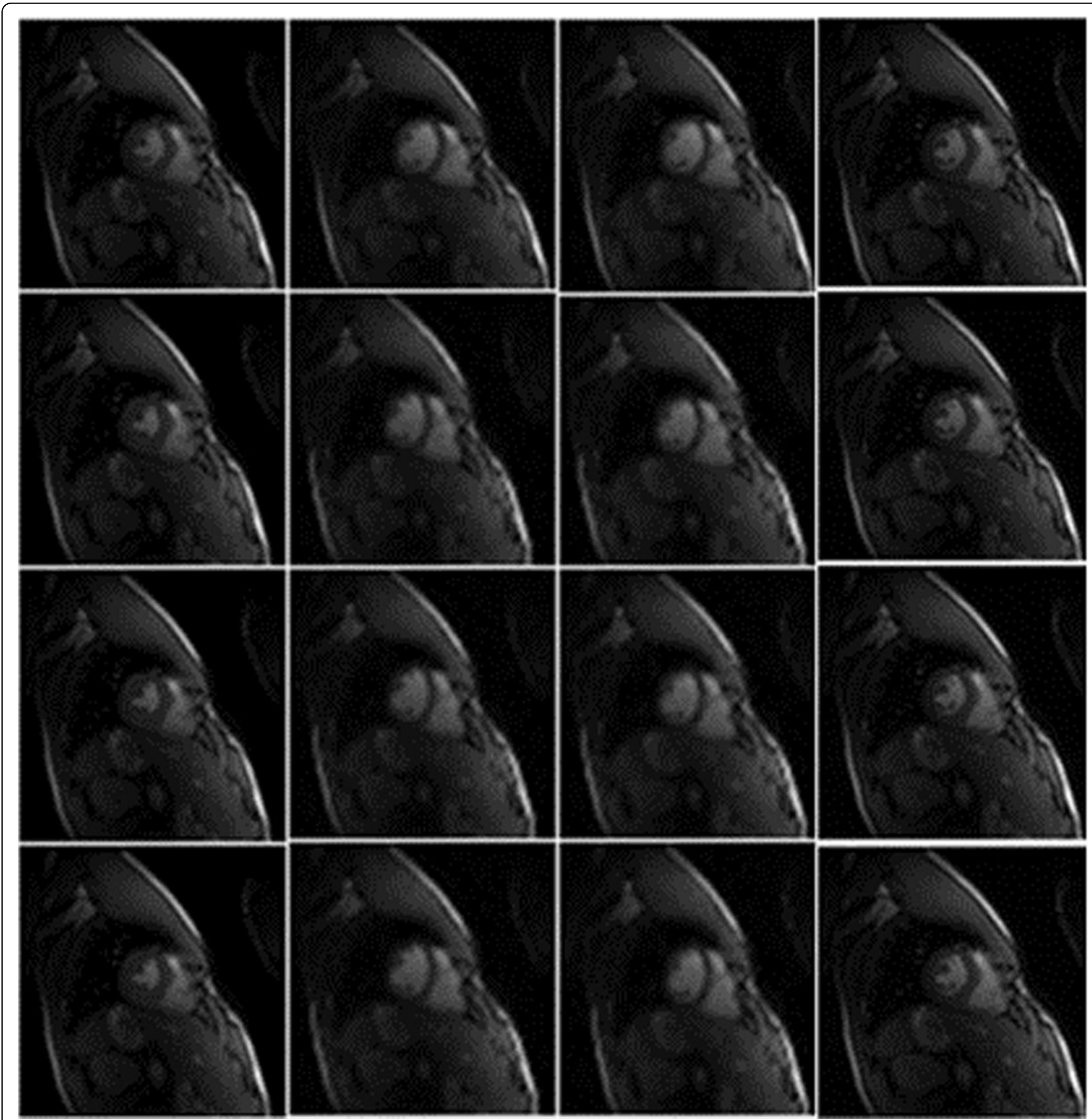

Figure 1 From left to right, First row) original image, CS frame with BWHT, BDCT and Gaussian measurement matrices. Second row) CS-TI using I method frame with BWHT, BDCT and Gaussian measurement matrices. Third row) CS-TI using FME method frame with BWHT, BDCT and Gaussian measurement matrices. Fourth row) CS-TI using (F-B) ME method frame with BWHT, BDCT and Gaussian measurement matrices.

Table 1 Results Of Proposed Methods Show That BWHT Outperform Other Methods.

\begin{tabular}{cccccccc}
\hline Measurement Matrix & methods & TI - Methods & SNR & PSNR & SSIM & Time(s) \\
\hline & & & & 26.750626 & 82.80 & 0.981008 & 11.3048 \\
\cline { 2 - 7 } & \multirow{3}{*}{ BWHT } & CS-TI & I & 28.076336 & 83.82 & 0.983096 & 2.5165 \\
\cline { 3 - 7 } & & & FME & 27.796233 & 83.88 & 0.974924 & 2.1939 \\
\hline
\end{tabular}


Table 1 Results Of Proposed Methods Show That BWHT Outperform Other Methods. (Continued)

\begin{tabular}{|c|c|c|c|c|c|c|}
\hline & CS & & 21.545575 & 75.25 & 0.827131 & 12.1182 \\
\hline & & I & 22.505806 & 76.34 & 0.780809 & 2.9337 \\
\hline \multirow[t]{4}{*}{ Gaussian } & CS-TI & FME & 20.527749 & 72.95 & 0.791594 & 2.9910 \\
\hline & & F-B ME & 21.586516 & 76.32 & 0.805057 & 3.0172 \\
\hline & CS & & 26.985696 & 82.07 & 0.974128 & 12.6598 \\
\hline & & 1 & 27.656441 & 83.16 & 0.958932 & 2.8911 \\
\hline \multirow[t]{2}{*}{$\mathrm{BDCT}$} & CS-TI & FME & 27.720514 & 83.87 & 0.977975 & 2.3867 \\
\hline & & F-B ME & 29.923755 & 83.86 & 0.962291 & 2.1828 \\
\hline
\end{tabular}

\section{Author details}

${ }^{1}$ Biomedical Engineering, Amirkabir University of Technology (Tehran

Polytechnic ), Tehran, Islamic Republic of Iran. ${ }^{2}$ David Geffen School of Medicine, UCLA, Los Angeles, CA, USA. ${ }^{3}$ Medical Physics and Biomedical Engineering, Tehran University of Medical Sciences, Tehran, Islamic Republic of Iran. ${ }^{4}$ Quantitative MR Imaging and Spectroscopy Group, Research Center for Molecular and Cellular Imaging, Tehran University of Medical Sciences, Tehran, Islamic Republic of Iran.

Published: 30 January 2013

\section{Reference}

1. Bilen C, Wang Y, Selesnick I: High Speed Compressed Sensing Reconstruction in Dynamic Parallel MRI Using Augmented Lagrangian and Parallel Processing. 2012, arXiv preprint arXiv: 1203.4587.

- Convenient online submission

- Thorough peer review

- No space constraints or color figure charges

- Immediate publication on acceptance

- Inclusion in PubMed, CAS, Scopus and Google Scholar

- Research which is freely available for redistribution 